\title{
Maximum Likelihood Estimation from Interval-Valued Data. Application to Fuzzy Clustering
}

\author{
Hani Hamdan ${ }^{(\bowtie)}$ \\ Laboratoire des Signaux et Systèmes, CentraleSupélec, CNRS, \\ Université Paris-Sud, Université Paris-Saclay, Paris, France \\ Hani.Hamdan@centralesupelec.fr
}

\begin{abstract}
Interval-valued data are used in many applications where they represent data imprecision, measurement inaccuracy, or measurand variability. As a result of the increasing use of such data in data mining, many data analysis methods have been extended to interval data this last decade. The ExpectationMaximization (EM) algorithm has been widely used for maximum likelihood estimation of parameters in statistical models, where the model depends on unobserved latent variables. In our keynote talk, we will present the EM algorithm to interval-valued data. In this contribution, we provide an original likelihood expression for interval data. Then, we propose an original method to introduce the imprecision and the variability of data into the mathematical expectation of the EM algorithm. The maximization of the obtained expectation gives place to the EM algorithm for interval-valued data. We apply this EM algorithm to mixture model for maximum likelihood estimation of mixture model parameters from interval-valued data. A special attention is paid for the case of Gaussian mixture models. In order to show the usefulness of our approach, we apply it on real interval-valued data issued from a flaw diagnosis application using acoustic emission.
\end{abstract}

